\title{
Vaterschaftsurlaub, what else?
}

Zum Schweizerischen Reformbedarf im Lichte der Markin Rechtsprechung des Europäischen Gerichtshofs für Menschenrechte

Irene Grohsmann*

Sowohl gemäss der Rechtsprechung des Europäischen Gerichtshofs für Menschenrechte (EGMR) als auch gestützt auf die Schweizerische Bundesverfassung dürfen Frau und Mann nicht aufgrund traditioneller Rollenverteilungen und Geschlechterstereotypen unterschiedlich behandelt werden. Seit den 1990er Jahren hat ein gesellschaftlicher Wandel stattgefunden, welcher die Rolle des Vaters stärkt. Dies hat auch in den Rechtsordnungen der Mehrzahl der europäischen Staaten und der Rechtsprechung des EGMR Niederschlag gefunden. Die aktuelle Regelung in der Schweiz, wonach nur Mütter einen bezahlten Urlaub nach der Geburt eines Kindes erhalten können, beruht auf traditionellen Vorstellungen über die Rolle von Frauen als Betreuerinnen der Kinder und Männer als Ernährer. Diese sind vor dem Hintergrund des gesellschaftlichen Wandels in Europa und der Schweiz nicht mehr tragbar.

I. Einleitung .33

II. Warum Vaterschaftsurlaub?

1. Gleichstellungspolitische Gründe - Neuordnung der traditionellen Rollenverteilung.34

2. Beitrag zur besseren Vereinbarkeit von Familie und Beruf .34

III. Der Fall Markin gegen Russland - Europäischer Gerichtshof für Menschenrechte .35

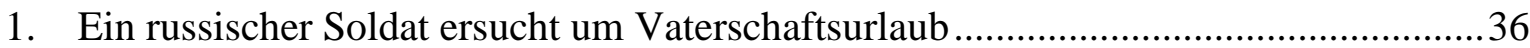

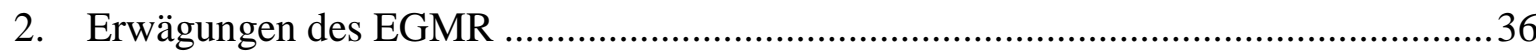

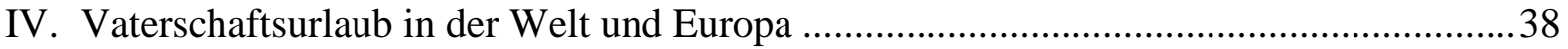

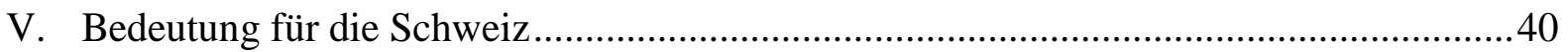

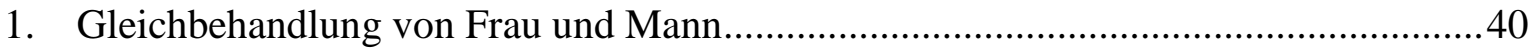

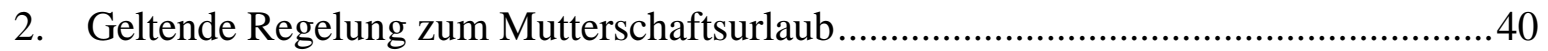

3. Geltende Regelung zum Vaterschaftsurlaub? ............................................................ 41

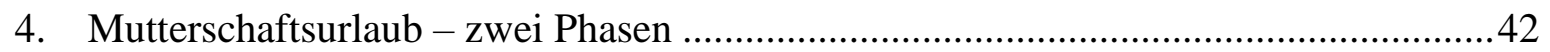

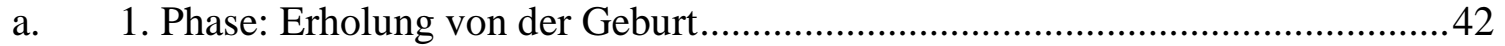

b. 2. Phase: Aufbau der Bindung mit dem Kind und Organisation Familienalltag ... 42

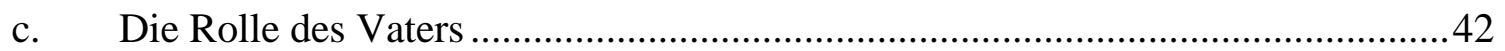

d. Auswirkungen auf die Organisation des Familienlebens ....................................43

5. Positive Entwicklungen für ein Umdenken in der Schweiz.........................................44

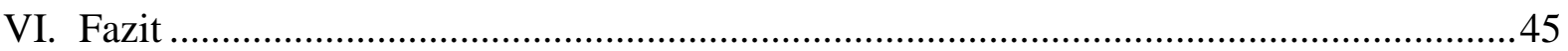

Zitiervorschlag: Irene Grohsmann, Vaterschaftsurlaub, what else?, in: sui-generis 2014, S. 33

URL: $\quad$ sui-generis.ch/3

DOI: $\quad$ https://doi.org/10.21257/sg.3 


\section{Einleitung}

1 In der Schweiz steht Vätern nach der Geburt ihres Kindes bekanntlich kein gesetzlich geregelter Vaterschaftsurlaub ${ }^{1}$ zur Verfügung. Vielmehr dürfen Väter zur Geburt ihres Kindes, gleich wie z.B. für einen Umzug, bloss einen bis zwei übliche freie Tage nach Art. 329 Abs. $3 \mathrm{OR}^{2}$ beziehen. Solche übliche freie und bezahlte Tage stehen allen Arbeitnehmenden zur Erledigung von persönlichen Angelegenheiten oder bei Familienereignissen (z.B. Hochzeit, Beerdigung) zu. Diese Zeit ist für einen Umzug oder eine Hochzeitsfeier durchaus angemessen, finden diese Ereignisse doch nach ein bis zwei Tagen einen Abschluss. Anderes müsste nach der Geburt eines Kindes gelten. Obwohl die Geburt an sich nach einigen Stunden vorbei ist, beginnt erst danach eine intensive Zeit für Vater und Mutter. Die Rechtsordnung anerkennt dies bisher nur für die Mutter und geht erstens davon aus, dass diese in den ersten Wochen das Kind hauptsächlich alleine betreuen muss und eine soziale Bindung mit dem Kind aufbauen soll und zweitens, dass der Vater kein vergleichbares Interesse hat.
2 Diese Einschätzung beruht auf der traditionellen Rollenverteilung zwischen Frau und Mann; diese gilt heute als überholt. Immer mehr Männer, Frauen, Väter und Mütter gehen heute davon aus, dass die Kinderbetreuung nicht alleine Aufgabe der Mutter sein soll. Laut einer Studie eines grossen Partnervermittlungsportals zu Beginn dieses Jahres befürworten $73 \%$ der 1'004 befragten Personen einen gesetzlich verankerten und bezahlten Vaterschaftsurlaub. Einen unbezahlten Vaterschaftsurlaub können sich 59\% der Befragten vorstellen, während $41 \%$ angaben, sich unbezahlten Vaterschaftsurlaub schlicht nicht leisten zu können ${ }^{3}$.

3 Der Europäische Gerichtshof für Menschenrechte (EGMR) hat in einem Leiturteil $2012^{4}$ festgehalten, dass Väter und Mütter nicht gestützt auf Geschlechterstereotypen unterschiedliche Rechte erhalten dürfen und ihnen unter denselben Voraussetzungen Urlaub zur Betreuung ihrer Kinder gewährt werden muss. Dieser Beitrag befasst sich mit den Auswir-
* MLaw, Vorstandsmitglied des Vereins gleichbeRICHTIG, Wissenschaftliche Mitarbeiterin im Themenbereich Geschlechterpolitik am Schweizerischen Kompetenzzentrum für Menschenrechte SKMR.

Die Autorin dankt Prof. Dr. Regula Kägi-Diener herzlich für die Zurverfügungstellung der Beschwerdeschrift an das Schweizerische Bundesgericht in Sachen X. gegen die Ausgleichskasse des Kantons Bern.

Dieses Werk ist lizenziert unter einer Creative Commons Namensnennung - Weitergabe unter gleichen Bedingungen 4.0 International Lizenz.

1 Zur Terminologie: Der Vaterschaftsurlaub ist ein individuelles Recht des Vaters. Er ist in der Regel direkt an die Geburt eines Kindes oder die Aufnahme eines Kindes zur Adoption gebunden und soll es dem Vater ermöglichen, in den ersten Wochen nach der Geburt oder der Ankunft des Kindes in seiner neuen Familie beim Kind und der Mutter zu bleiben. Der Elternurlaub kann von beiden Elternteilen beansprucht werden. Er beinhaltet einerseits den geburtsgebundenen Mutterschaftsurlaub und gibt beiden Eltern andererseits die Möglichkeit, gleichberechtigt in den ersten Lebensmonaten oder Jahren ihres Kindes für die Pflege, Betreuung und Erziehung zuständig zu sein. Elternurlaub kann für den
Vater reservierte Zeit enthalten. Siehe dazu: Vaterschaftsurlaub und Elternurlaub. Auslegeordnung und Präsentation unterschiedlicher Modelle. Bericht des Bundesrates in Erfüllung des Postulats Fetz (11.3492) vom 6. Juni 2011, 30. Oktober 2013, S. 2. In Übereinstimmung mit der Eidgenössischen Koordinationskommission für Familienfragen (EKFF) wären die Bezeichnungen «Vaterschaftszeit», «Elternzeit» und, in diesem Zusammenhang, auch «Mutterschaftszeit», passender. $\mathrm{Da}$ in der rechtlichen, politischen und gesellschaftlichen Diskussion jedoch von «Urlaub» gesprochen wird, verwendet auch dieser Artikel diese Terminologie.

2 Bundesgesetz betreffend die Ergänzung des Schweizerischen Zivilgesetzbuches (Fünfter Teil: Obligationenrecht), (OR; SR 220).

3 Studie Parship.ch, Medienmitteilung vom 18.02.2014: «Ob bezahlt oder unbezahlt: Die meisten Männer befürworten den Vaterschaftsurlaub.» Siehe auch die Kampagne von Travail Suisse:Höchste Zeit für einen Vaterschaftsurlaub.

4 Urteil 3008/06 des Europäischen Gerichtshofs für Menschenrechte (EGMR) vom 20. März 2012 (Markin gegen Russland). 
kungen, welche dieses Urteil auf die Schweizerische Rechtsordnung haben kann und zeigt auf, dass sowohl die politischen als auch rechtlichen Vorzeichen in der Schweiz für eine Einführung eines Vaterschaftsurlaubs gut stehen.

\section{Warum Vaterschaftsurlaub?}

4 Studien gehen davon aus, dass ein Zusammenhang besteht zwischen Vaterschaftsurlaub, der Übernahme von Familienarbeit durch Männer und der Entwicklung eines Kindes. Väter, welche unmittelbar nach der Geburt ihres Kindes, insbesondere während zwei oder mehr Wochen, eine berufliche Auszeit nehmen, sind auch später mehr in das Leben ihrer Kinder involviert. Dies kann insbesondere positive Auswirkungen auf die Geschlechtergleichstellung zu Hause und damit auch auf die Geschlechtergleichstellung im Erwerbsleben haben ${ }^{5}$ :

«By drawing fathers into the daily realities of childcare, free of workplace constraints, extended time off [immediately after the birth] provides the space necessary for fathers to develop the parenting skills and sense of responsibility that then allows them to be active co-parents rather than helpers to their female partners. This shift from a manager-helper dynamic to that of co-parenting creates the opportunity for the development of a more gender-equitable division of labour. ${ }^{, 6}$

\section{Gleichstellungspolitische Gründe - Neu- ordnung der traditionellen Rollenvertei- lung}

5 Annahmen, dass Männer auf die Rolle des Ernährers der Familie festgelegt sind, sind ausserordentlich hartnäckig und führen dazu, dass die Rolle des Mannes als Vater und Betreuer seiner Kinder ignoriert wird. Für Väter, die sich gerne mehr um ihre Kinder kümmern möchten, stellt diese Rollenverteilung ein grosses Hindernis dar. Gestützt auf die Prämisse, die Wertschätzung von Männern hänge von ihrer Leistung im Erwerbsleben ab, stehen für Männer einerseits ausserordentlich wenige Möglichkeiten zur Verfügung, sich neben dem Erwerbsleben auch in das Familienleben einzugliedern. Andererseits führt sie dazu, dass die bestehenden Möglichkeiten vermehrt von Frauen wahrgenommen werden und die Hürde für Männer damit noch grösser wird, orientieren sich diese doch in ihren Entscheidungen an ihren männlichen Kollegen, Verwandten und Vorgesetzten (sog. «sozialer Schneeballeffekt»). Ein gesetzlich verankerter bezahlter Vaterschaftsurlaub gibt Vätern einen allgemein anerkannten und gesellschaftlich akzeptierten Anspruch auf Achtung ihrer familiären Interessen und kann zu einer egalitären Aufteilung der Familien- und Hausarbeit und damit zum Abbau von geschlechterstereotypen Rollenbildern beitragen ${ }^{7}$.

\section{Beitrag zur besseren Vereinbarkeit von Familie und Beruf}

6 Der Bundesrat bestätigte in seinem Bericht vom 30. Oktober 2013 zum Vaterschafts- und Elternurlaub $^{8}$, dass Vaterschaftsurlaub positive Auswirkungen auf die Gleichstellung im Erwerbsleben haben kann:

«Es hat sich gezeigt, dass ein solcher Urlaub
zu einer partnerschaftlicheren Rollenteilung
in der Familie beitragen kann, indem er der
Mutter und dem Vater bereits unmittelbar
nach der Geburt die Möglichkeit eröffnet,
sich intensiv an der Betreuung und Erziehung

Vol. 28 No. 1, Februar 2014, 110-132, S. 110.

7 Bericht des Bundesrates zum Vaterschaftsurlaub und Elternurlaub (FN 1), S. 26.

8 Bericht des Bundesrates zum Vaterschaftsurlaub und Elternurlaub (FN 1), S. 66. 
des Kindes sowie der Hausarbeit zu beteiligen. Beide Eltern können ihre familiären Aufgaben wahrnehmen, ohne dass sie gezwungen werden, ihre Erwerbstätigkeit zugunsten der Familie aufzugeben. Somit zählt der Vaterschafts- respektive Elternurlaub zu den Massnahmen, welche die Vereinbarkeit von Familie und Erwerbstätigkeit für junge $\mathrm{Fa}$ milien zu verbessern vermögen.»"

7 Dies zahlt sich auch für Arbeitgebende (finanziell) aus. Unternehmen, die heute freiwillig Vaterschaftsurlaub gewähren, berichten von mehr Zufriedenheit und weniger Krankeitsabwesenheiten und Fluktuation der Mitarbeitenden ${ }^{10}$.

\section{Der Fall Markin gegen Russland - Euro- päischer Gerichtshof für Menschenrechte}

8 Der Europäische Gerichtshof für Menschenrechte (EGMR) hat sich in zwei ${ }^{11}$ Entscheiden mit dem Thema Vaterschaftsurlaub befasst. Die eingereichten Beschwerden stützten sich jeweils auf das Diskriminierungsverbot (Art. 14 EMRK $^{12}$ ) und das Recht auf Familienleben, welches durch Art. 8 EMRK geschützt ist. Während der EGMR 1998 im Urteil Petrovic gegen Österreich ${ }^{13}$ die Klage eines Vaters auf Beanspruchung der nur Frauen zustehenden finanziellen Unterstützung für den Karenzurlaub noch abgewiesen hatte, änderte er im Lichte der Entwicklungen in den Europaratsstaaten seine Rechtsprechung.

9 Herr Petrovic hatte nach der Geburt seines Kindes im Februar 1989 Antrag auf sog. «Karenzurlaubsgeld» gestellt, da er, und nicht seine Frau, die Karenz in Anspruch nahm. Sein Gesuch wurde abgewiesen mit der Begründung, das Karenzurlaubsgeld stehe nur der Mutter zu. Der mit dem Fall befasste EGMR führte damals aus, dass eine unterschiedliche Behandlung von Frauen und Männern aufgrund des Geschlechts vorliege. Der Fall Petrovic spielte sich jedoch Ende der 1980er Jahre ab. Damals bestand im Hinblick auf gesetzliche Regelungen der bezahlten Karenz in den Konventionsstaaten noch keine weitgehende Übereinstimmung. Entsprechend führte der Gerichtshof zu dieser Zeit aus, dass die Ablehnung des Antrags auf Gewährung von Karenzurlaubsgeld im Bereich des staatlichen Ermessenspielraums lag, welcher den Konventionsstaaten zur Beurteilung der Frage, ob und in welchem Umfang Unterschiede in ansonsten ähnlichen Situationen eine unterschiedliche rechtliche Behandlung rechtfertigen, eingeräumt wird. Damit stellte der EGMR am 27. März 1998 keine Verletzung von Art. 14 i.V.m. Art. 8 EMRK fest ${ }^{14}$.

10 Am 20. März 2012 urteile der Gerichtshof im Fall Markin gegen Russland ${ }^{15}$, dass Regelungen, durch welche Frauen und Männer unterschiedlich behandelt werden, nicht auf Geschlechterstereotypen basieren dürfen. Staaten, die einen Elternurlaub vorsehen, müssen diesen Mutter und Vater unter den gleichen Voraussetzungen gewähren. Der Ermessensspielraum der Staaten ist damit heute enger als noch zur Zeit des Petrovic Urteils.
9 Bericht des Bundesrates zum Vaterschaftsurlaub und Elternurlaub (FN 1), S. 66.

10 Eidgenössisches Büro für die Gleichstellung von Frau und Mann EGB, Best Practices; siehe auch von Bresinski, Bernhard, Die Zukunft der Schweizer Geschlechter- und Familienpolitik, in: Männerzeitung 1_2011.

11 Siehe ausserdem den hängigen Fall Hallier und Lucas gegen Frankreich (Nr. 46386/10) betreffend dem An- trag einer Frau in eingetragener Partnerschaft auf Elternurlaub wegen der Geburt des Kindes ihrer eingetragenen Partnerin.

12 Konvention zum Schutze der Menschenrechte und Grundfreiheiten (EMRK; SR 0.101).

13 Urteil 20458/92 des Europäischen Gerichtshofs für Menschenrechte (EGMR) vom 27. März 1998 (Petrovic gegen Österreich).

14 EGMR, Petrovic gegen Österreich (FN 13), § 30-43.

15 EGMR, Markin gegen Russland (FN 4). 


\section{Ein russischer Soldat ersucht um Vater- schaftsurlaub}

11 In der Sache ging es um Konstantin Markin, ein Angehöriger der russischen Armee. Am 30. September 2005 gebar seine Ehefrau ihr drittes gemeinsames Kind. Gleichentags wurde die Ehe geschieden und Herr Markin übernahm das alleinige Sorgerecht für alle drei Kinder. Am 11. Oktober 2005 ersuchte Herr Markin beim Leiter seiner militärischen Abteilung um die Gewährung von drei Jahren Elternurlaub, welcher in Russland gemäss Art. 255 und 256 des russischen Arbeitsgesetzes von 2001 Frauen und Männern zusteht. Sein Gesuch wurde jedoch abgelehnt mit der Begründung, dass ein dreijähriger Elternurlaub nur weiblichen Armeeangehörigen zustehe. Männliche Soldaten erhielten grundsätzlich keinen Vaterschaftsurlaub. Wenn die Mutter des Kindes bei der Geburt sterbe oder der Vater für ein Kind unter 14 Jahren alleine verantwortlich sei (z.B. weil die Mutter verstorben ist oder der Vater das alleinige Sorgerecht erhalten hat), könne ihnen ein Urlaub von drei Monaten zugesprochen werden, um die Betreuung der Kinder zu organisieren oder um zu entscheiden, aus der Armee auszutreten. Männliche Armeeangehörige würden insofern sowohl anders als weibliche Armeeangehörige, als auch anders als die männliche Zivilbevölkerung behandelt ${ }^{16}$.

12 Gegen diesen Bescheid erhob Herr Markin innerstaatlich Beschwerde und brachte vor, die Weigerung, ihm drei Jahre Vaterschaftsurlaub zu gewähren, verletze den verfassungsmässigen Grundsatz der Gleichbehandlung von Frau und Mann. Nach Durchlaufen des inner-

16 EGMR, Markin gegen Russland (FN 4), § 9-33.

17 EGMR, Markin gegen Russland (FN 4), § 9-33.

18 EGMR, Markin gegen Russland (FN 4), § 76, § 102108. staatlichen Instanzenzugs gelangte er schliesslich am 21. Mai 2006 an den EGMR ${ }^{17}$ und rügte eine Verletzung von Art. 14 EMRK (Diskriminierungsverbot) i.V.m. Art. 8 EMRK (Recht auf Privat- und Familienleben). Als männlicher Angehöriger des Militärs stehe ihm der Bezug eines dreijährigen Elternurlaubs nicht zu, während dieser weiblichen Angehörigen des Militärs gewährt würde. Diese Regelung beruhe auf einer geschlechterstereotypen Rollenaufteilung, die Männern die Aufgabe des Versorgers und Frauen die Aufgabe der Kinderbetreuerin zuweise. Damit würden sowohl die Väter (in Bezug auf ihr Familienleben) als auch die Mütter (in Bezug auf ihr Erwerbsleben) benachteiligt. Die Weigerung, ihm gestützt auf sein Geschlecht den Bezug von Urlaub zur Betreuung seines Kindes zu gewähren, könne nicht mit objektiven Gründen gerechtfertigt werden und stelle daher eine Diskriminierung dar ${ }^{18}$. Damit könne dem Einwand Russlands, Frauen werde im Zusammenhang mit Mutterschaft eine «besondere soziale Rolle» ${ }^{19}$ zu teil, welche die Ungleichbehandlung rechtfertige, nicht gefolgt werden.

\section{Erwägungen des EGMR}

\section{a. Schutzbereich Familienleben Art. 8 EMRK}

13 Gemäss Art. 8 EMRK hat jede Person das Recht auf Achtung ihres Familienlebens. Geschützt sind tatsächlich gepflegte Beziehungen innerhalb der Kernfamilie (Eltern, Kinder), unter Umständen auch weitere Beziehungen (z.B. zu den Grosseltern). Die Bestimmung begründet ein Recht auf Zusammenleben und auf persönliche Kontakte unter den Familienmitgliedern $^{20}$.

19 EGMR, Markin gegen Russland (FN 4), § 34.

20 Grabenwarter, Christoph, Kommentar zu Art. 8, Rn 19 ff., in: European Convention on Human Rights, Commentary, C.H. Beck, München, 2014. 
Gerichtshof führte aus, dass aus dem Recht auf Familienleben keine positive Leistungspflicht des Staates fliesse, bezahlten Elternurlaub zur Verfügung $\mathrm{zu}$ stellen. Ein Recht auf Elternurlaub könne daher aus Art. 8 EMRK nicht abgeleitet werden. Dennoch sei Art. 8 EMRK auf Elternurlaub anwendbar: Werde einem Elternteil die Möglichkeit eines Urlaubs gegeben, habe dieser gewährte Elternurlaub und die entsprechenden finanziellen Beiträge Auswirkungen auf das Familienleben und beeinflusse zwangsläufig dessen Organisation. Wenn ein Staat Elternurlaub vorsehe, müsse dieser Frauen und Männern diskriminierungsfrei gewährt werden ${ }^{21}$.

\section{b. Diskriminierungsverbot Art. 14 EMRK}

Frauen und Männer als Kinderbetreuer in vergleichbarer Situation

Laut dem Gerichtshof befänden sich Männer und Frauen im Zusammenhang mit Elternurlaub in vergleichbaren Situationen. Es müsse unterschieden werden zwischen der Zeit nach der Geburt, welche die Frau biologisch bedingt zur Erholung von den Strapazen der Geburt benötige und der Zeit, welche beiden Elternteilen zur Pflege und Herstellung einer persönlichen Beziehung mit dem Kind diene. In dieser zweiten Phase könne nicht mehr mit biologischen Gründen argumentiert werden, sondern Frau und Mann seien in ihrer Rolle als Betreuungspersonen des Kindes einander gleichgestellt («similarly placed») ${ }^{22}$. Im vorliegenden Fall handle es sich daher um Beschränkungen des Rechts auf Privat- und Familienlebens des Vaters, welche nur durch objektive und vernünftige Gründe gerechtfertigt werden können ${ }^{23}$.
Gesellschaftliche Entwicklung hin zum Elternurlaub

16 Bezugnehmend auf das Argument Russlands, auch andere Europaratsstaaten gewährten männlichen Angehörigen der Armee keinen Elternurlaub, verwies der EGMR auf sein Urteil Petrovic gegen Österreich aus dem Jahre 1998 und die Entwicklung, welche seither in Europa stattgefunden habe. 1998 befand der EGMR noch, dass keine einheitliche europäische Praxis betreffend der Regelung von Elternurlaub für Väter auszumachen sein, da in einer Mehrzahl der Europaratsstaaten der Bezug von bezahltem Elternurlaub für Väter nicht vorgesehen war. Entsprechend sei der Ermessensspielraum der Staaten grösser gewesen als bei Vorliegen eines allgemeinen Konsenses. Seither hätten sich die sozialen Gegebenheiten und mit ihr die Rechtsordnungen der Mehrheit der europäischen Staaten jedoch gewandelt. Die Rechtsordnungen der meisten Europaratsstaaten gewährten heute sowohl Frauen als auch Männern den Bezug von Elternurlaub, während Staaten, die Elternurlaub nur für Frauen vorsehen, in der Minderheit seien. Gleiches gelte im Übrigen für Angehörige der Armee. Diese Entwicklung zeige, dass sich die europäischen Gesellschaften in Richtung ausgeglichener Teilung der Verantwortlichkeit für die Betreuung von Kindern bewegen und die Rolle von Männern als Betreuer an Anerkennung gewonnen habe. Diese umfassende und fortlaufende Entwicklung könne der Gerichtshof nicht ignorieren ${ }^{24}$.

Traditionelle Rollenbilder keine Rechtfertigung

17 Die unterschiedliche Behandlung von Frauen und Männern könne ausserdem nicht durch Berufung auf traditionelle Rollenbilder und Geschlechterstereotypen gerechtfertigt wer-
21 EGMR, Markin gegen Russland (FN 4), § 130.

EGMR, Markin gegen Russland (FN 4), § 131/132.

EGMR, Markin gegen Russland (FN 4), § 133-137.
Siehe dazu auch Grabenwarter (FN 20), Kommentar zu Art. 14, Rn. 4 ff.

24 EGMR, Markin gegen Russland (FN 4), § 140. 
den. Gleich wie Regelungen gestützt auf Stereotypen betreffend Herkunft, sexuelle Orientierung oder Hautfarbe seien Ungleichbehandlungen von Frauen und Männern, die nur auf der Vorstellung des Mannes als Haupternährer der Familie und der Frau als Betreuerin der Kinder beruhen, nicht zulässig. Der Gerichtshof äusserte hier ausdrücklich seine Ansicht, dass diese Unterscheidungen dazu beitrügen, die Existenz von Geschlechterstereotypen weiterzuführen, was sowohl negative Auswirkungen auf die Karriere von Frauen als auch auf das Familienleben von Männern habe ${ }^{25}$.

\section{c. Verletzung von Art. 14 EMRK i.V.m. Art. 8 EMRK}

18 Der Gerichtshof kam mit 16 gegen eine Stimme zum Schluss, dass die traditionelle Rollenverteilung zwischen den Geschlechtern in der Gesellschaft nicht dazu dienen könne, den Ausschluss der Männer, inklusive jener, die in der Armee dienten, vom Recht auf Elternurlaub zu rechtfertigen. Damit verletze die gesetzliche Regelung Russlands, nur weiblichen Angehörigen der Armee den Bezug eines dreijährigen Elternurlaubs zu gewähren, das Recht auf Familienleben des Beschwerdeführers wegen Diskriminierung aufgrund seines
Geschlechts (Verletzung von Art. 14 EMRK in Verbindung mit Art. 8 EMRK) ${ }^{26}$.

\section{Vaterschaftsurlaub in der Welt und Eu- ropa}

19 Bezahlter Vaterschaftsurlaub hat heute in 71 Ländern der Welt ${ }^{27}$ eine gesetzliche Grundlage. In Afrika gewähren 28 Staaten $^{28}$ den Vätern einen bezahlten Urlaub von einem bis 15 Tagen, in Asien kennen sechs Staaten ${ }^{29}$ einen bezahlten Vaterschaftsurlaub von einem bis 10 Tage. In Osteuropa und Zentralasien steht Vätern in drei Staaten ${ }^{30}$ bezahlter Urlaub von einem bis mindestens 16 Tagen zu, in Mittelund Südamerika und der Karibik kann in 11 Staaten bezahlter Vaterschaftsurlaub von einem Tag bis zwei Wochen bezogen werden ${ }^{31}$. Im Mittleren Osten kennt nur ein Staat $^{32}$ einen bezahlten Vaterschaftstag. In Westeuropa, Nordamerika und Australien/Neuseeland gewähren 22 Staaten $^{33}$ mit einem bis zu mehr als 16 bezahlten Tagen einen Vaterschaftsurlaub.

20 Die meisten europäischen Staaten kennen bezahlten Vaterschaftsurlaub von mehr als zwei Tagen und/oder bezahlten Elternurlaub ${ }^{34}$ :
25 EGMR, Markin gegen Russland (FN 4), § 141-143.

26 EGMR, Markin gegen Russland (FN 4), § 150-152.

27 Siehe ILO, Maternity and Paternity at Work (FN 5), S. 150 ff. Daten sind von 167 der insgesamt 193 Staaten der Welt verfügbar.

28 Algerien, Benin, Burkina Faso, Burundi, Kamerun, Zentralafrikanische Republik, Chad, Komoren, Kondo, Elfenbeinküste, Demokratische Republik Kongo, Dschibuti, Gabun, Kenia, Libyen, Madagaskar, Mali, Mauretanien, Mauritius, Marokko, Mozambique, Ruanda, Seychellen, Südafrika, Tansania, Togo, Tunesien, Uganda. Äthiopien (unbezahlt).

29 Bangladesch, Kambodscha, Indonesien, Myanmar, Philippinen, Singapur. Südkorea (unbezahlt).

30 Bosnien und Herzegowina, Kroatien, Serbien. Aserbaidschan und Kasachstan (unbezahlt).
31 Argentinien, Brasilien, Chile, Kolumbien, Dominikanische Republik, Ecuador, El Salvador, Guatemala, Paraguay, Uruguay, Venezuela. Bahamas (unbezahlt).

32 Saudi Arabien. Syrien (unbezahlt).

33 Australien, Belgien, Bulgarien, Dänemark, Estland, Finnland, Frankreich, Griechenland, Ungarn, Island, Italien, Lettland, Litauen, Luxemburg, Niederlande, Polen, Portugal, Rumänien, Slowenien, Spanien, Schweden, Grossbritannien. Norwegen und Neuseeland (unbezahlt).

34 Siehe ILO, Maternity and Paternity at Work (FN 5), S. 150 ff. und OECD, Social Policy Division, Directorate of Employment, Labour and Social Affairs, Parental leave systems, 1. Mai 2014, S. 2. 


\begin{tabular}{|c|c|c|c|c|}
\hline Land & $\begin{array}{l}\text { Dauer Vater- } \\
\text { schaftsurlaub }\end{array}$ & $\begin{array}{l}\text { Bezahlung in \% } \\
\text { des Lohns }\end{array}$ & $\begin{array}{l}\text { Dauer Elt- } \\
\text { ernurlaub }^{35}\end{array}$ & $\begin{array}{l}\text { Bezahlung in \% } \\
\text { des Lohns }\end{array}$ \\
\hline Belgien & 10 Tage & $\begin{array}{l}100 \% \text { erste } 3 \text { Ta- } \\
\text { ge, danach } 82 \%{ }^{36}\end{array}$ & 17 Wochen & Pauschalbetrag \\
\hline Dänemark & 2 Wochen & $100 \%$ & 32 Wochen & $100 \%$ \\
\hline Deutschland & - & - & 52 Wochen & $67 \%$ \\
\hline Finnland & 54 Tage & $70 \%$ & 25 Wochen & $70 \%$ \\
\hline Frankreich & 11 Tage & $100 \%$ & 26 Wochen & Pauschalbetrag \\
\hline Grossbritannien & 2 Wochen & $90 \%$ & 13 Wochen & Unbezahlt \\
\hline Island & 13 Wochen & $80 \%$ & 13 Wochen & $80 \%$ \\
\hline Italien & $1 \mathrm{Tag}$ & $100 \%$ & 26 Wochen & $30 \%$ \\
\hline Luxemburg & 2 Tage & $100 \%$ & 26 Wochen & Pauschalbetrag \\
\hline Norwegen & 14 Tage & unbezahlt & $49-59 \mathrm{Wo}^{37}$ & $80-100 \%$ \\
\hline Österreich & - & - & 104 Wochen & Pauschalbetrag \\
\hline Polen & 14 Tage & $100 \%$ & 104 Wochen & $\begin{array}{l}60 \% \text { während } \\
26 \text { Wochen }\end{array}$ \\
\hline Portugal & 20 Tage & $100 \%$ & $\begin{array}{l}\text { 17-21 } \\
\text { Wochen }\end{array}$ & $80-100 \%$ \\
\hline Schweden & 10 Tage & $80 \%$ & $\begin{array}{l}80 \\
\text { Wochen }^{39}\end{array}$ & $\begin{array}{l}80 \% \text { während } 65 \\
\text { Wochen }^{40}\end{array}$ \\
\hline Spanien & 15 Tage & $100 \%$ & 156 Wochen & Unbezahlt \\
\hline Tschechien & - & - & 155 Wochen & Pauschalbetrag \\
\hline Ungarn & 5 Tage & $100 \%$ & 104 Wochen & $70 \%$ \\
\hline Schweiz & - & - & - & - \\
\hline USA & - & - & 12 Wochen & Unbezahlt \\
\hline Türkei & - & - & - & - \\
\hline Russland & - & - & 156 Wochen & $\begin{array}{l}40 \% \text { während } \\
\text { ersten } 78 \text { Wo }\end{array}$ \\
\hline
\end{tabular}

Tabelle 1, gestützt auf Daten der ILO und der OECD.

21 Auch der EGMR hatte im Rahmen des Markin Urteils eine vergleichende Studie der Gesetzgebungen von 33 Europaratssaaten vorgenommen. Er kam zu dem Schluss, dass 28
Staaten sowohl Urlaub für Väter als auch Elternurlaub vorsehen, welche wie gesehen unterschiedlich ausgestaltet sein können ${ }^{41}$.

35 Für Vater und Mutter zur Verfügung stehender Urlaub.

$36 \quad 100 \%$ erste 3 Tage, danach $82 \%$

37 Jeweils 14 Wochen reserviert für den Vater bzw. die Mutter.

38 Danach Pauschalbetrag.

39 Davon müssen 480 Tage von den Eltern geteilt werden.

40 Danach Pauschalbetrag.

41 EGMR, Markin gegen Russland (FN 4), § 71-75. 


\section{Bedeutung für die Schweiz}

\section{Gleichbehandlung von Frau und Mann}

22 Wie die Europäische Menschenrechtskonvention kennt die Schweizerische Bundesverfassung ein Verbot der Diskriminierung aufgrund des Geschlechts (Art. 8 Abs. 2 BV $^{42}$ ). Ausserdem verpflichtet Art. 8 Abs. $3 \mathrm{BV}$ alle staatlichen Behörden, Mann und Frau gleichberechtigt zu behandeln und neben der rechtlichen auch für die soziale Gleichstellung der Geschlechter zu sorgen ${ }^{43}$. Damit ergibt sich die staatliche Aufgabe, tatsächliche Gleichstellung in der sozialen Wirklichkeit zu schaf$f^{4}{ }^{44}$. Gemäss bundesgerichtlicher Rechtsprechung müssen Frau und Mann in allen Bereichen gleich behandelt werden, ohne Rücksicht auf gesellschaftliche Verhältnisse und Vorstellungen. Differenzierungen durch die Rechtsordnung gestützt auf das Geschlecht sind grundsätzlich unzulässig ${ }^{45}$. Zulässig ist «eine unterschiedliche Behandlung von Mann und Frau nur noch dann, wenn auf dem Geschlecht beruhende biologische oder funktionale Unterschiede eine Gleichbehandlung absolut ausschliessen. ${ }^{46}$ Wegen dem in Art. 8 Abs. 3 BV verankerten Gleichbehandlungsgebot können namentlich traditionelle Rollenverteilungen nicht als Rechtfertigung für eine Ungleichbehandlung herangezogen werden ${ }^{47}$.

Ähnlich wie der EGMR im Urteil Markin gegen Russland verbietet damit auch die schwei-

42 Bundesverfassung der Schweizerischen Eidgenossenschaft (BV; SR 101).

43 Bigler-Eggenberger, Margrith, Kommentar zu Art. 8 Abs. 3 BV, in: Die schweizerische Bundesverfassung, Kommentar, 2. Auflage, Zürich, St. Gallen, Basel, Genf, 2008, S. 211-226, Rn. 75/75.

44 BGE 125 I 21, E. 3a.

45 BGE 129 I 265, E. 3.2.

46 BGE 129 I 265, E. 3.2; BGE 126 I 1E. 2c; BGE 125 I 21, E. 3a; BGE 123 I 56 E. 2b; BGE 120 V 312 E. $2 a$.

47 BGE 129 I 265, E. 3.3.

4801.426 - Revision Erwerbsersatzgesetz. Ausweitung zerische Rechtsordnung eine ungleiche Behandlung von Frau und Mann gestützt auf traditionelle Geschlechterrollen und Geschlechterstereotypen und lässt geschlechtsbezogene Regelungen nur im absoluten Ausnahmefall $\mathrm{zu}$, wenn biologische Unterschiede keine andere Wahl lassen.

\section{Geltende Regelung zum Mutterschaftsur- laub}

24 In der Schweiz wurde am 1. Januar 2005 die Mutterschaftsentschädigung und damit ein bezahlter Mutterschaftsurlaub eingeführt. Nachdem 2002 die Parlamentarische Initiative von Pierre Tripone $\mathrm{z}^{48}$ gutgeheissen wurde, nahm das schweizerische Stimmvolk in der Referendumsabstimmung vom 26. September 2004 die Änderung des Bundesgesetz über den Erwerbsersatz für Dienstleistende und bei Mutterschaft ${ }^{49}$ mit $55.4 \%$ Ja-Stimmen $a^{50}$. Der Mutterschaftsurlaub ist seither in Art. 329f OR sowie im Erwerbsersatzgesetz gesetzlich verankert.

25 Gemäss Art. 16b ff. EOG hat jede berufstätige $^{51}$ und AHV-versicherte Mutter Anspruch auf den Bezug eines Taggeldes in der Höhe von $80 \%$ ihres letzten Erwerbseinkommens ${ }^{52}$. Der Anspruch beginnt am Tag der Niederkunft und endet am 98. Tag nach seinem Beginn. Stirbt die Frau vor Ablauf dieser Zeit oder nimmt sie ihre Erwerbstätigkeit wieder auf, endet der Anspruch vorzeitig.

der Erwerbsersatzansprüche auf erwerbstätige Mütter.

49 Erwerbsersatzgesetz (EOG; SR 834.1).

50 01.426 Parlamentarische Initiative Revision Erwerbsersatzgesetz. Ausweitung der Erwerbsersatzansprüche auf erwerbstätige Mütter. Bericht der Kommission für soziale Sicherheit und Gesundheit des Nationalrates, 3. Oktober 2002, BB1 2002 7522; Bundesamt für Sozialversicherungen.

51 Während der neun Monate unmittelbar vor der Niederkunft muss sie mindestens fünf Monate lang eine Erwerbstätigkeit ausgeübt haben.

52 Bzw. maximal 196 Franken pro Tag. 


\section{Geltende Regelung zum Vaterschaftsurlaub}

26 Ein dem Mutterschaftsurlaub entsprechendes Recht steht Vätern nicht zu. Gesetzlich geregelt ist eine ein- oder zweitägige bezahlte Auszeit von der Erwerbstätigkeit; dies im Rahmen der «üblichen freien Tage» nach Art. 329 Abs. 3 OR. Privaten Arbeitgebenden steht es jedoch frei, im Rahmen ihrer Möglichkeiten bezahlten Vaterschaftsurlaub zu gewähren. Einige (grosse) Arbeitgeber gewähren ihren Angestellten heute freiwillig bis $\mathrm{zu} 10$ Tage Vaterschaftsurlaub. Eine einheitliche und gleiche Behandlung privatrechtlich angestellter Väter besteht aber nicht.

27 Die meisten Staatsangestellten können bereits heute von besseren Bedingungen profitieren als privatrechtlich Beschäftigte. Auf Bundesebene besteht für das Personal der Bundesverwaltung mit Art. $17 \mathrm{a} \mathrm{BPG}^{53}$ und Art. 40 Abs. 3 lit. b VBPV ${ }^{54}$ eine gesetzliche Grundlage für den Vaterschaftsurlaub ${ }^{55}$. Damit gibt es heute in der Schweiz grosse Unterschiede in der Gewährung eines Vaterschaftsurlaubs, je nach Arbeitgeber und Region:

\section{Privater Sektor}

\begin{tabular}{|l|l|l|l|}
\hline Arbeitgeber & $\begin{array}{l}\text { Anzahl Arbeit- } \\
\text { nehmende }\end{array}$ & $\begin{array}{l}\text { Dauer Vater- } \\
\text { schaftsurlaub }\end{array}$ & $\begin{array}{l}\text { Bezahlung in \% } \\
\text { des Lohns }\end{array}$ \\
\hline Credit Suisse & $19^{`} 400$ & 14 Tage & $100 \%$ \\
\hline Roche & $11^{`} 700$ & 6 Tage & $100 \%$ \\
\hline Migros & $8^{`} 200$ & 14 Tage & $100 \%$ \\
\hline UBS & $22^{`} 229$ & 10 Tage & $100 \%$ \\
\hline Swisscom & $19^{`} 400$ & 10 Tage & $100 \%$ \\
\hline \multicolumn{2}{|r}{ Tabelle 2 $^{56}$}
\end{tabular}

\section{Öffentlicher Sektor}

\begin{tabular}{|c|c|c|c|}
\hline Arbeitgeber & $\begin{array}{l}\text { Kanton / Ge- } \\
\text { meinde }\end{array}$ & $\begin{array}{l}\text { Vater- } \\
\text { schaftsurlaub }\end{array}$ & $\begin{array}{l}\text { Bezahlung in \% } \\
\text { des Lohns }\end{array}$ \\
\hline $\begin{array}{l}\text { Bundesver- } \\
\text { waltung }\end{array}$ & - & 10 Tage & $100 \%$ \\
\hline \multirow{5}{*}{$\begin{array}{l}\text { Kantonale } \\
\text { Verwaltung }\end{array}$} & $\begin{array}{l}\mathrm{BE}^{57}, \mathrm{GL}, \mathrm{SO}, \\
\mathrm{AT}, \mathrm{AI}, \mathrm{TG}, \mathrm{OW}\end{array}$ & 1 bis 2 Tage & $100 \%$ \\
\hline & $\begin{array}{l}\text { UR, SZ, GR, } \\
\mathrm{AG}^{58}\end{array}$ & 3 bis 4 Tage & $100 \%$ \\
\hline & $\begin{array}{l}\text { ZH, NW, ZG, FR, } \\
\text { BL }^{59}, \text { SH, SG, } \\
\text { TI, VD, VS, NE }\end{array}$ & 5 Tage & $100 \%$ \\
\hline & GE, BS ${ }^{60}$ & 10 Tage & $100 \%$ \\
\hline & $\mathrm{JU}$ & 12 Tage & $100 \%$ \\
\hline \multirow{5}{*}{$\begin{array}{l}\text { Kommunale } \\
\text { Verwaltung }\end{array}$} & $\begin{array}{l}\text { Basel, St.Gallen, } \\
\text { Lugano, Biel }\end{array}$ & 5 Tage & $100 \%$ \\
\hline & $\begin{array}{l}\text { Zürich, Winter- } \\
\text { thur, Luzern }\end{array}$ & 10 Tage & $100 \%$ \\
\hline & Genf 61 & 4 Wochen & $100 \%$ \\
\hline & Lausanne & 21 Tage & $100 \%$ \\
\hline & Bern & 3 Wochen & $100 \%$ \\
\hline
\end{tabular}

53 BPG; SR 172.220.1.

54 Verordnung des EFD zur Bundespersonalverordnung (VBPV;SR 172.220.111.31).

55 Botschaft zu einer Änderung des Bundespersonalgesetzes, 31. August 2011, BB1 2013 6703, S. 6717.

56 Grossen, Joel und Brindley, Callum, Comparative Study of National Paternity Leave Policies, Seminar on Gender and Sustainability, University of Bern, IZFG, 2014, S. 5.

57 Art. 156 Abs. 1 lit. b Personalverordnung, Kanton Bern, Nr. 153.011.1.
58 § 33 Abs. 1 lit. c Personal- und Lohnverordnung, Kanton Aargau, Nr. 165.111.

$59 \S 11$ Verordnung über den Elternurlaub, Kanton BaselLandschaft, Nr. 153.13.

$60 \$ 18$ Abs. 1 Ziff. 3 Ferien- und Urlaubsverordnung Kanton Basel-Stadt, Nr. 162.410.

61 Art. 70 Statut du personnel de la Ville de Genève, Nr. LC 21151.

62 Bericht des Bundesrates zum Vaterschaftsurlaub und Elternurlaub (FN 1), S. 14/15. 


\section{Mutterschaftsurlaub - zwei Phasen}

Das Erwerbsersatzgesetz gibt Frauen einen Anspruch auf Urlaub unmittelbar nach der Geburt ihres Kindes. Männer sind von dieser Regelung ausgeschlossen, ihnen steht ein vergleichbarer Anspruch nicht zu. Wie gesehen sind solche auf dem Geschlecht basierende Ungleichbehandlungen nur zulässig, wenn sie auf zwingenden biologischen oder faktischen Unterschieden beruhen, welche eine Ungleichbehandlung erfordern. Insbesondere darf nicht auf eine traditionelle Rollenverteilung abgestellt werden. Auch im Hinblick auf die Ausführungen des EGMR, Frauen und Männer befinden sich bei der Betreuung ihrer Kinder in vergleichbaren Situationen ist es fraglich, ob tatsächlich genügende biologische Gründe bestehen, um nur Frauen einen Urlaub nach der Geburt eines Kindes zu gewähren.

Der heute bestehende Mutterschaftsurlaub von 14 Wochen ist aufgeteilt in zwei Phasen: Während die ersten acht Wochen aus biologischen Gründen zwingend gewährt werden müssen, dienen die übrigen sechs Wochen dem Aufbau einer sozialen Bindung mit dem Neugeborenen sowie der Organisation des Familienalltags.

\section{a. Erste Phase: Erholung von der Geburt}

Die Beschränkung auf die Frau beruht während der ersten acht Wochen nach der Niederkunft auf zwingend biologischen Gründen (Stichwort Wochenbett). Während dieser Zeit bilden sich die schwangerschafts- und geburtsbedingten Veränderungen des weiblichen Körpers zurück. Entsprechend sieht auch Art. 35a Abs. 3 des Arbeitsgesetzes ${ }^{63}$ zur Erholung der sog. «Wöchnerinnen» ein gesund- heitspolizeiliches Arbeitsverbot von acht Wochen vor.

\section{b. Zweite Phase: Aufbau der Bindung mit dem Kind und Organisation Familienall- tag}

30 Von der 9.-14. Woche hingegen wird der Urlaubs- und Entschädigungsanspruch der Frau nicht mehr aus zwingend biologischen Gründen, sondern aus sozialen Überlegungen gewährt: Die Frau soll sich unbesorgt dem Kind widmen können und im Hinblick auf ihren beruflichen Wiedereinstieg das Familienleben und die Kinderbetreuung organisieren können. Gemäss der Stellungnahme des Bundesrates zur Einführung der Mutterschaftsversicherung soll demnach «der Mutter [...] eine Ruhepause nach der Niederkunft ermöglicht werden, damit sie sich erholen und möglichst unbelastet für das Neugeborene sorgen und ihre Beziehung zu ihm aufbauen kann» ${ }^{64}$. Es steht jeder Mutter jedoch frei, nach der achten Woche auf den weiteren Urlaubsanspruch zu verzichten und ihre Erwerbstätigkeit wieder aufzunehmen, wodurch sie den Entschädigungsanspruch verliert (Art. 35a Abs. 3 ArG und Art. 16d EOG).

\section{c. Die Rolle des Vaters}

31 Dass es nun zwischen der 9.-14. Woche alleine Sache der Frau sein soll, sich um das Kind zu kümmern, beruht nicht auf biologischen Gründen, sondern auf traditionellen gesellschaftlichen Vorstellungen der Aufgaben von Frauen und Männern in der Familie und verkennt die Rolle des Vaters. Auch der Vater muss und darf eine soziale Beziehung zu seinem Kind aufbauen. Dies ist nicht nur ein

63 Bundesgesetz über die Arbeit in Industrie, Gewerbe und Handel (ArG; SR 822.11).

64 Stellungnahme des Bundesrates vom 6. November 2002 zu 01.426 Parlamentarische Initiative Revision

Erwerbsersatzgesetz. Ausweitung der Erwerbsersatzansprüche auf erwerbstätige Mütter (Triponez Pierre). Bericht vom 3. Oktober 2002 der Kommission für soziale Sicherheit und Gesundheit des Nationalrates, BB1 2003 1112, S. 1114. 
Wunschanliegen der sog. «neuen Väter», sondern ein rechtlich gestütztes Interesse, sieht doch das Schweizerische Zivilgesetzbuch $\left(Z_{G B}{ }^{65}\right)$ ein partnerschaftliches Verhältnis der Eltern vor und differenziert nicht zwischen den Rechten der Mütter und Väter (Art. 270 ff. ZGB $)^{66}$. Durch die heutige Regelung wird das Interesse der Väter gestützt auf ihr Geschlecht unzulässig beeinträchtigt.

Insofern muss nun von der Einschätzung des Bundesverwaltungsgerichts im Jahre 1994 abgewichen werden. Das Gericht hatte damals, vor Einführung der Mutterschaftsversicherung auf Bundesebene, in Abweisung einer Beschwerde eines Vaters betreffend seines Gesuchs um Gewährung eines bezahlten Vaterschaftsurlaubs von 14 Wochen argumentiert, dass neben den ersten acht Wochen des Mutterschaftsurlaubs auch die letzten sechs Wochen auf biologischen Gründen basieren:

«Wie schon dargelegt, lässt sich ein Mutterschaftsurlaub in der vom Regierungsrat [des Kantons Bern] vorgesehenen Dauer mit einer grosszügig bemessenen Erholungszeit nach der Niederkunft sowie mit dem Stillen des Neugeborenen rechtfertigen. Daran ändert nichts, dass der Regierungsrat mit der Ausdehnung des Mutterschaftsurlaubs [von 8 auf 14 Wochen] auch das sozialpolitische Ziel verfolgen wollte, der Mutter die Doppelrolle in Familie und Beruf zu erleichtern. Die Motive einer gesetzlichen Regelung lassen sich häufig nicht auseinanderhalten. Für die Vereinbarkeit mit Art. 4 Abs. 2 BV genügt, dass objektiv biologische Unterschiede zwischen den Geschlechtern bestehen, welche eine rechtliche Differenzierung zu tragen vermögen.» ${ }^{67}$
33 Es drängt sich ausserdem die Frage auf, ob der Zweck der ersten acht Wochen des Mutterschaftsurlaubs, nämlich die Erholung und Regeneration der Mutter, ohne die Hilfe des Vaters erreicht werden kann. Da biologisch bedingt die Frauen die Schwangerschaft und die Geburt übernehmen, benötigen Männer nach der Geburt keine Erholungszeit. Soll sich jedoch die Frau von der Geburt erholen können, benötigt sie zwingend Hilfe. Es sollte ausser Frage stehen, dass der Vater des Kindes diese Hilfe leisten muss, schulden sich Vater, Mutter und Kind doch gegenseitigen Beistand (Art. 273 ZGB). Dazu brauchen Väter die Möglichkeit, vom Arbeitsplatz abwesend zu sein. Darüber verfügen aktuell jedoch nur Väter, welche sich dies finanziell leisten können. Dass in den ersten acht Wochen ein gewisses Bedürfnis nach Unterstützung besteht, zeigt nicht zuletzt das Angebot der Spitex, während des Wochenbetts eine Haushaltshilfe zur Verfügung zu stellen ${ }^{68}$.

\section{d. Auswirkungen auf die Organisation des Familienlebens}

34 Wie der EGMR in seinen Entscheiden zum Vaterschaftsurlaub festgestellt hat, hat die Gewährung oder Verweigerung von Urlaub nach der Geburt eines Kindes Einfluss auf die aktuelle und zukünftige Organisation des Familienlebens. Hier ist ein zentraler Ansatzpunkt zur Verwirklichung der Geschlechtergleichstellung auszumachen und Regelungen, welche diese Organisation gestützt auf traditionelle Rollenaufteilungen beeinflussen, dürfen entsprechend nicht mehr zulässig sein. Bisher führt der ausschliessliche Urlaubsanspruch der Mutter noch zwangsläufig dazu, dass sie von Beginn an die Aufgabe der Kinderbetreuung übernimmt und damit die traditionelle
65 Schweizerisches Zivilgesetzbuch, (ZGB; SR. 210).

66 Kägi-Diener, Regula, Beschwerdeschrift Bundesgericht in Sachen X. gegen Ausgleichskasse Kanton Bern, 20. November 2013 (liegt der Autorin vor).
67 Urteil des Bundesverwaltungsgerichts vom 11. Februar 1994 i.S. X., BVR 1995 S. 109-116, S. 116.

68 Spitex bei Mutterschaft, siehe auchForum Geburt und der Erfahrungsbericht einer Mutter. 
Rollenaufteilung perpetuiert wird. Zurzeit werden Frauen tatsächlich dazu gezwungen, sich hauptsächlich um die familiären Angelegenheiten zu kümmern, da sich in den Wochen des Mutterschaftsurlaubs bereits die Zuständigkeiten einpendeln. Aufgrund der starren Ordnung der Arbeitswelt lassen sich diese Zuständigkeiten im Nachhinein nicht mehr oder nur schwer ändern. Während Frauen damit stets in die Rolle der Familienbetreuerin gedrängt werden, werden Väter aus dieser Rolle herausgedrängt.

\section{Positive Entwicklungen für ein Umden- ken in der Schweiz}

Obwohl in den vergangenen zehn Jahren 26 Parlamentarische Vorstösse ${ }^{69}$ zur Einführung eines Vaterschafts- oder Elternurlaubs gescheitert sind, zeigen diese konstant anhaltenden Bemühungen, dass in weiten Kreisen ein politischer Wille zur Änderung besteht. Auch die Einführung der Mutterschaftsversicherung wurde im Parlament und an der Urne mehrmals abgelehnt, bis sie schliesslich 2004 angenommen wurde. Dies macht deutlich, dass das Überwinden von traditionellen Geschlechterstereotypen nicht einfach ist und durchaus Zeit und Diskussion benötigt. Dieses Jahr sind im Parlament bisher drei ${ }^{70}$ Vorstösse zum Thema bezahlter Vaterschaftsurlaub/Elternurlaub eingegangen. Sogar die Interpellation Frehner ${ }^{71}$, die die Auswirkungen der Beziehungen zwischen der Schweiz und der EU auf das Sozialsystem der Schweiz zum Thema hat, identifiziert die Einführung eines Vaterschafts- o- der Elternurlaubs als eine von sieben in diesem Zusammenhang relevanten Fragen. Dies zeigt das Gewicht dieses Themas in der politischen Arena von links bis rechts.

Der Bericht des Bundesrates kann bereits als erster Fortschritt betrachtet werden, wurden bisher doch die parlamentarischen Vorstösse bereits im Keim erstickt. Die Kommission für soziale Sicherheit und Gesundheit des Ständerates (SGK-S) hat angekündigt, sich Anfang 2015 grundsätzlich über die Strategie des Bundesrates im Bereich der Familienpolitik orientieren zu lassen ${ }^{72}$. Dabei wird auch die fehlende bundesrätliche Priorisierung des Vaterschafts- oder Elternurlaubes zur besseren Vereinbarkeit von Familie und Beruf diskutiert werden müssen. Denn wie der EGMR ausgeführt hat, betrifft die Gewährung oder Verweigerung von Elternurlaub die Organisation des Familienlebens von Anfang an und damit eben gerade nicht «nur die begrenzte Zeit nach der Geburt des Kindes ${ }^{73}$. Mütter würden auch mittel- bis langfristig entlastet und die Vereinbarkeit von Beruf und Familie erleichtert. Im Zusammenhang mit der Umsetzung der Volksinitiative «Gegen Masseneinwanderung» könnte der Bundesrat allenfalls früher als gedacht wieder mit dem Thema Vaterschaftsurlaub befasst werden. Laut seinem Konzept zur Umsetzung der Initiative müsse das einheimische Arbeitskräftepotenzial erhöht werden, damit der Bedarf des Arbeitsmarktes vermehrt im Inland gedeckt werden könne. In einer Verbesserung der Vereinbarkeit von Familie und Beruf liege dabei ein
69 Bericht des Bundesrates zum Vaterschaftsurlaub und Elternurlaub (FN 1), S. 73 ff. (Anhang 2: Parlamentarische Vorstösse auf Bundesebene zum Vaterschaftsurlaub bzw. Elternurlaub).

70 Motion Grünliberale Fraktion (14.3068) Elternurlaub statt Mutterschaftsentschädigung; Motion Andrea Caroni (14.3109) Elternurlaub. Mehr Wahlfreiheit bei gleichen Kosten; Parlamentarische Initiative Martin
Candinas, (14.415) Zwei Wochen über die EO bezahlten Vaterschaftsurlaub; Interpellation Sebastian Frehner (14.3483) Rechtsübernahme bei der Sozialwerken.

71 Interpellation Sebastian Frehner (14.3483) Rechtsübernahme bei der Sozialwerken.

72 Medienmitteilung SGK-S vom 16. Mai 2014.

73 Bericht des Bundesrates zum Vaterschaftsurlaub und Elternurlaub (FN 1), S. 66. 
grosses Potenzial ${ }^{74}$. Es gibt daher momentan mehr als genug Anlass, das Thema Vaterschaftsurlaub prioritär anzugehen.

\section{Fazit}

37 Im oben dargestellten Urteil des EGMR machte dieser deutlich, dass aus Art. 8 EMRK keine Verpflichtung für Staaten abgeleitet werden kann, einen Vaterschafts- oder Elternurlaub einzuführen. Er wies allerdings auch darauf hin, dass unterschiedliche Regeln für Frauen und Männer nicht auf traditionellen Rollenbildern und Geschlechterstereotypen basieren dürfen. Ausserdem schützt Art. 8 EMRK das Recht der Eltern, eine ausreichende Beziehung zu ihren Kindern zu begründen und zu pflegen. In der Schweiz wird dies der Mutter heute zugestanden, dem Vater jedoch nicht. Darin liegt die Ungleichbehandlung respektive Diskriminierung des Vaters, welche auch mit der Schweizerischen Bundesverfassung nicht vereinbar ist. Geschlechterstereotype Überlegungen rechtfertigen nicht die unterschiedliche Behandlung von Frau und Mann bzw. eine Abweichung von der Gleichstellung von Frau und Mann nach Art. 8 Abs. 3 Satz 1 BV und Art. 14 EMRK.

Wie gezeigt liegen der heute in der Schweiz geltenden Regelung traditionelle Aufgabenzuteilungen von Mutter und Vater zu Grunde. Zumindest in den letzten sechs Wochen des Mutterschaftsurlaubs sind aber keine geschlechtsbedingten biologischen/funktionalen Unterschiede mehr auszumachen, welche eine Gleichbehandlung absolut ausschliessen könnten. Der zwischen der 9.-14. Woche gesetzlich nur der Frau zugestandene Urlaubsund Entschädigungsanspruch diskriminiert daher die Männer. Um die heutige geschlechterstereotype Arbeitsverteilung zu durchbrechen, muss die Rechtsordnung angepasst werden und Vätern das gleiche Recht auf Urlaub zur Kinderbetreuung wie den Müttern gewährt werden. Die Möglichkeit, unbezahlten Vaterschaftsurlaub nehmen zu können, reicht dazu jedoch nicht aus. Vaterschaftsurlaub muss, gleich wie Mutterschaftsurlaub, für alle Väter möglich sein, die eine Auszeit für ihr Kind nehmen wollen und nicht nur diejenigen, die sich eine Auszeit finanziell leisten können.

39 Sowohl die Rechtsprechung des EGMR, die rechtliche und soziale Entwicklung in der Mehrheit der europäischen Länder als auch die gesellschaftliche und politische Diskussion in der Schweiz machen deutlich, dass die Schweizerische Rechtsordnung in Bezug auf die gleichberechtigte Betreuung eines Kindes durch seine Eltern hinterher hinkt. Im Interesse der Väter, Mütter und Kinder der Schweiz ist auf eine baldige Anpassung zu hoffen.

74 Schweizerische Eidgenossenschaft, Art. 121a BV (Steuerung der Zuwanderung) Umsetzungskonzept, Juni 2014, S. 21-23. 